\title{
Demonstration of a Rocking Prosthetic Mitral Valve Using 3-Dimentional Echocardiography and Live Surgical Imaging
}

\author{
Sean Baskin ${ }^{1}$, Rece Laney ${ }^{2}$, Senthil Nathan ${ }^{2}$, Feroze Mahmood ${ }^{1}$, and J. Michael Haering ${ }^{1}$ \\ ${ }^{1}$ Beth Israel Deaconess Medical Center Harvard Medical School \\ ${ }^{2}$ Beth Israel Deaconess Medical Center
}

July 29, 2021

\begin{abstract}
Prosthetic valve endocarditis is a complication of bacteremia which can cause damage to the prosthetic valve or the tissue to which it was sewn. Extensive tissue damage can result in a loss of anchoring and allow for abnormal valvular motion. Dehiscence can lead to excessive motion of the valve which is termed rocking. Through advances in imaging technology, live 3-dimentional (3-D) transesophageal echocardiography can allow for precise identification of the location of, and amount of dehiscence. We present a 37-year old male with a rocking prosthetic valve demonstrated on 3-D echocardiography and correlated to surgical manipulation.
\end{abstract}

Article Title: Demonstration of a Rocking Prosthetic Mitral Valve Using 3-Dimentional Echocardiography and Live Surgical Imaging. Sean M Baskin, DO, $\mathrm{MA}_{1}$; Rece Laney, MD, $\mathrm{MS}_{2}$; Venkatachalam Senthilnathan, $\mathrm{MD}_{2}$; Feroze Mahmood, MD, FASE 1 ; James Michael Haering $\mathrm{MD}_{1} 1$ : Department of Anesthesia, Critical Care and Pain Medicine. Beth Israel Deaconess Medical Center, Harvard Medical School; Boston, MA. 2: Department of Cardiovascular Surgery. Beth Israel Deaconess Medical Center, Boston, MA Name, mailing address, phone number, and e-mail address of the corresponding author. James Michael Haering, MD Feldberg 4401 Deaconess Road; CC-470; Boston, MA 02215 617-754-2101 Email: mhaering@bidmc.harvard.edu Financial Disclosures: None Conflicts of interest: None Abstract word count - 95

List each author's individual contribution to the manuscript:

Sean Baskin- This author prepared the manuscript and obtained the procedural video Rece Laney- This author edited the manuscript and provided surgical correlation Venkatachalam Senthilnathan- This author edited the manuscript and performed the surgery Feroze Mahmoud- This author obtained the echocardiographic images, and helped with manuscript editing. James Michael Haering- This author edited the manuscript

MeSH keywords:

Transesophageal echocardiography

Endocarditis

Mitral Valve Replacement

Dehiscence

Abstract:

Prosthetic valve endocarditis is a complication of bacteremia which can cause damage to the prosthetic valve or the tissue to which it was sewn. Extensive tissue damage can result in a loss of anchoring and allow for 
abnormal valvular motion. Dehiscence can lead to excessive motion of the valve which is termed rocking. Through advances in imaging technology, live 3-dimentional (3-D) transesophageal echocardiography can allow for precise identification of the location of, and amount of dehiscence. We present a 37-year old male with a rocking prosthetic valve demonstrated on 3-D echocardiography and correlated to surgical manipulation. This manuscript adheres to the applicable EQUATOR guidelines.

Glossary of Terms:

3 - $\mathrm{D}=3$-dimensional; 2 - $\mathrm{D}=2$-dimensional; $\mathrm{TEE}=$ transesophageal echocardiography; $\mathrm{MVR}=$ mitral valve replacement; MSSA=methicillin-susceptible Staphylococcus aureus; TTE=transthoracic echocardiography

Background:

Prosthetic valve endocarditis is a complication of bacteremia with destruction of the annular tissue and occurs in $0.1-1.3 \%$ of cases and can result in partial or complete prosthetic valvular dehiscence . Valvular dehiscence results in positional instability and the prosthetic valve demonstrates motion that is independent of the cardiac motion. The extent of this motion is determined by the degree of dehiscence of the prosthesis and ranges from subtle to frank "rocking" motion. Rocking motion of the valve implies extreme instability and impending dislodgment ${ }_{2}$. Diagnosis of prosthetic valve dehiscence requires demonstration of the valve ring motion independent of cardiac motion. Over the years, fluoroscopy has been replaced with two-dimensional (2D) echocardiography for assessment of prosthetic valve function and now threedimensional (3D) transesophageal echocardiography (TEE) has further enhanced our understanding. We present a case of dehiscence of a prosthetic valve in mitral position with the classic rocking motion as seen with live 3-dimensional transesophageal echocardiography (TEE). Written HIPAA authorization has been obtained from the patient.

Case Report

A 37year-old male with a prior mitral valve replacement due to infective endocarditis secondary to intravenous drug use presented with recurrent bacteremia and was found to have multiple prosthetic vegetations, (largest $14 \mathrm{~mm} \times 7 \mathrm{~mm}$ ) by echocardiography. Despite an antibiotic regimen and demonstrating clearance of bacteremia, he developed new onset shortness of breath and chest pain. A repeat TEE identified posterior dehiscence of the mitral valve prosthesis and he was admitted for urgent re-do sternotomy and redo MVR. On the day of surgery, after uneventful induction of general anesthesia an intraoperative TEE examination was performed using an Epiq Ultrasound System using an X7-2t TEE probe (Philips Medical Systems Andover MA). A comprehensive 2D examination was performed and the preoperative findings were confirmed (Figure 1). Posteriorly, there was a large area of valve dehiscence with communication between the left ventricle and left atrium (Figure 2). The prosthetic valve ring demonstrated significant independent motion. Next, an R-wave gated 3D full-volume acquisition was performed in the mid-esophageal position after optimizing the region of interest (prosthetic valve) in the lateral and elevational planes. After appropriate cropping and image adjustment, the "rocking" motion of the prosthetic valve could be visually appreciated from multiple perspectives (Video 1). It could also be visually appreciated that more than fifty percent of the circumference of the prosthetic valve ring had dehisced. This finding was also confirmed on direct surgical examination of the valve on cardiopulmonary bypass (video 2). The prosthetic valve was excised, and a $33 \mathrm{On}$-X mechanical mitral valve (NW Kennesaw GA USA) was placed. The rest of the clinical course was uneventful and he was subsequently discharged on the $11^{\text {th }}$ postoperative day to a rehabilitation facility.

Discussion

One of the earliest published descriptions of rocking motion, in 1979 by Schipira and colleagues described a hinge like motion of the partially secured aortic prosthesis as it changed position throughout the cardiac cycle. This motion was identified using 2-dimensional echocardiography and was compared with the expected normal motion of a well seated prosthesis ${ }_{3}$. Since then, rocking remains in the lexicon of echocardiographers to describe the prototypical movement of a prosthetic valve that has dehisced at least $50 \%$ from the annulus to which it was sewn. Clinical availability of $2 \mathrm{D}$ echocardiography marked a significant advancement over 
the use of m-mode echocardiography or fluoroscopy. 2D imaging allowed for better visualization and charac-

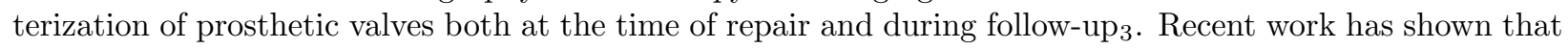
computerized tomography can have near $100 \%$ concurrence with surgical findings and be assistive in surgical planning $_{4}$. Availability of real-time 3D echocardiography has further enhanced our understanding of prosthetic valve function and pathology and prior authors have shown prosthetic aortic valvular pathology using 2-D and 3-D echocardiography ${ }_{5,6}$. We however present a mechanical mitral prosthesis where 3-dimentional echocardiography confirmed the requisite extent of dehiscence to allow for rocking motion.

Our case demonstrates that 3D imaging provides unique insight into the mechanism of "rocking" motion of a dehisced prosthetic valve. Also, the temporal sequence of events in our patient implies that development of dehiscence was associated with sudden appearance of symptoms and clinical deterioration. Based on the $3 \mathrm{D}$ imaging it can also be visually appreciated that there is more than $50 \%$ of circumferential dehiscence of the ring which provides the fulcrum and the hinge point of the rocking motion seen. This characteristic back and forth motion of the prosthetic ring generates greater mechanical sheering forces that ultimately may completely dislodge the prosthetic valve. Therefore, rapid progression of symptoms consistent with heart failure should raise the suspicion of dehiscence. As can be seen in videos 1 and 2, the extent of dehiscence identified was significant, and posed a significant risk for further detachment and catastrophic cardiovascular collapse. Initial 2-dimentional imaging provided initial assurance of valvular pathology, and 3-dimentional live imaging was utilized to establish the diagnosis, and to further identify the specific dehisced region of the mitral prosthesis. Real-time surgical imaging as seen in video 2 confirmed the echocardiographic findings.

Conclusion:

New onset symptoms consistent with regurgitant valvular lesions should prompt significant concern from providers, especially in patients with pre-existing valvular prostheses as this could signify partial dehiscence. When dehiscence exceeds $50 \%$ of the attached circumference, rocking motion is highly probable. Rocking motion represents excessive motion of the prosthesis independent of the annulus and can lead to catastrophic sequela. Echocardiographers should promptly assess any areas of paravalvular regurgitation to evaluate the region, extent and severity of detachment. 3-Dimentional TEE may provide highly useful images for both diagnosis and possible interventional planning. If rocking motion is identified, prompt surgical intervention should be considered.

\section{References:}

1. Baddour LM et al for the Committee on Rheumatic Fever, Endocarditis, and Kawasaki Disease., Council on Cardiovascular Disease in the Young., Councils on Clinical Cardiology, Stroke, and Cardiovascular Surgery and Anesthesia., American Heart Association., Infectious Diseases Society of America. Circulation. 2005 Jun 14; 111(23):e394-434

2. Tominaga R, Kurisu K, Ochiai Y, Tomita Y, Masuda M, Morita S, Yasui H. A 10-year experience with the Carbomedics Cardiac Prosthesis. Ann Thorac Surg. 2005; 79: 784-789

3. Schapira, N; Martin RP; Fowles, RE; et al. Two dimensional echocardiographic assessment of patients with bioprosthetic valves. Am J Cardiol, 1979;43(3)510-519

4. Tsai IC, Lin YK, Chang Y, Fu YC, Wang CC, Hsieh SR, Wei HJ, Tsai HW, Jan SL, Wang KY, Chen MC, Chen CC. Correctness of multi-detector-row computed tomography for diagnosing mechanical prosthetic heart valve disorders using operative findings as a gold standard. Eur Radiol. 2009 Apr;19(4):857-67.

5. Gonzalez-Ferrer JJ, Fernandez-Jimenez R, Perez de Isla L, Rocafort AG, Zamorano JL. Rocking Aortic Valve Prosthesis: A 3-Dimentional Transesophageal Echocardiography View. J Am Coll Cardiol. 2010 Apr, 55(17) e135

6. Gerstein NS, Bhamidipati CM, Schulman PM. Near-Complete aortic mechanical valve dehiscence due to endocarditis reinfection. Echocardiography 2018 Jun; 35(6):872-875

7. Figures: Figure 1: Midesophageal 4-chamber view in mid-systole showing rocking of the posterior portion of the mitral prosthesis during ventricular systole. LA: Left Atrium, LV: Left Ventricle. RA: Right Atrium. Arrow: Area of prosthetic mitral valve dehiscence. Star: Prosthetic mitral valve oriface 
Figure 2: Mid-esophageal Long Axis in mid-systole showing severe regurgitation through the posterior dehiscence of the mitral prostethesis. Ao: Ascending Aorta LV: Left ventricle. RV: Right ventricle. Star: Area of prosthetic dehiscence. Arrows (multiple): Flow of regurgitant jet through the area of dehiscence Videos: Video 1: 3-D reconstruction of rocking motion Video 2: Side-by-side of 3-D reconstruction showing rocking motion with direct surgical visualization.
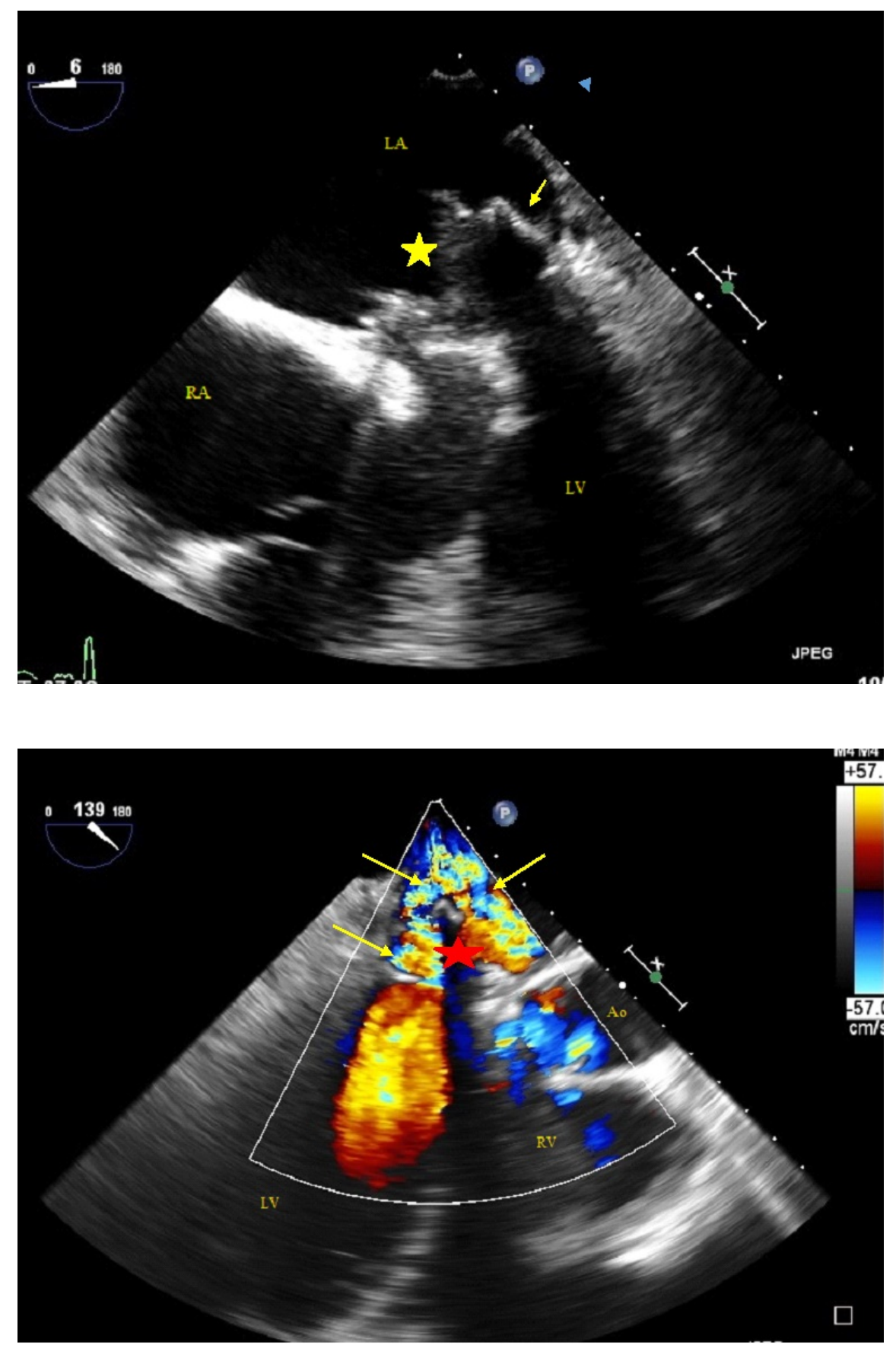